\title{
Escala CARE de empatia: tradução para o Português falado no Brasil e resultados iniciais de validação
}

\section{CARE scale of empaty: translation-to the Portuguese spoken in Brazil and initial validation results}

Giuliano Roberto Scarpellini', Gabriel Capellato', Fabíola Galhardo Rizzatti ${ }^{2}$, Geruza Alves da Silva ${ }^{3}$, José Antonio Baddini-Martinez ${ }^{3}$

\section{RESUMO}

Objetivos: Traduzir e adaptar para uso no Brasil a escala de avaliação de empatia clínica Consultation and Relational Empathy (CARE). Fornecer resultados preliminares de validação concorrente dessa escala.

Métodos: A escala em inglês foi traduzida e adaptada seguindo metodologia recomendada. Vinte pacientes atendidos em ambulatório de pneumologia em hospital público ligado ao Sistema Único de Saúde foram entrevistados quanto ao grau de compreensão da versão final do instrumento. Em uma segunda fase, doze pacientes do mesmo ambulatório responderam a versão brasileira da escala CARE e a Escala de Percepção de Empatia Pelo Paciente (EPEP), logo após término de consulta de rotina. Os médicos responsáveis pelo atendimento desses pacientes também foram convidados, após a consulta, a responder questionário contendo a versão brasileira do Interpersonal Reactivity Index (IRI) e o Inventário de Empatia (IE).

Resultados: Dezenove dos 20 pacientes referiram grau elevado de compreensão dos itens da versão brasileira da escala CARE. O escore mediano da escala CARE para outros 12 voluntários foi 44,5 (2063). O coeficiente alfa de Cronbach para as respostas dos últimos foi 0,867 . Os escores CARE correlacionaram-se de maneira significante com os escores EPEP $(r=0,699 ; p=0,01)$ e com o subitem altruísmo do IE $(r=0,744, p=0,01)$. A escala CARE não mostrou correlações significantes com os escores globais das medidas de empatia IRI e IE informados pelos médicos.

Conclusão: A versão brasileira da escala CARE é de fácil compreensão e exibe evidências aceitáveis de validade concorrente e consistência interna. Medidas de empatia referidas pelos médicos tendem a não se correlacionar com as percepções dos pacientes.

Palavras chave: Empatia. Psicologia Médica. Escalas de Valor Relativo. Tradução.

1. Acadêmicos de Medicina da Faculdade de Medicina de Ribeirão Preto da Universidade de São Paulo

2. Professora Adjunta do Departamento de Medicina do Centro de Ciências Biológicas e da Saúde da Universidade Federal de São Carlos

3. Professores Associados do Departamento de Clínica Médica da Faculdade de Medicina de Ribeirão Preto da Universidade de São Paulo
Correspondência: José Baddini-Martinez Avenida Bandeirantes, 3900, CEP; 14048-900 - Ribeirão Preto, SP baddini@fmrp.usp.br

Artigo recebido em 30/08/2013 Aprovado para publicação em 24/01/2014 


\section{Introdução}

O contato diário com pacientes é elemento central da atividade médica. Vínculos de comunicação efetiva precisam ser estabelecidos, não apenas entre o médico e os seus pacientes, como também entre ele e familiares, amigos e cuidadores. Nesse contexto, cada vez mais vem sendo dada importância à empatia como instrumento essencial da relação profissional da saúde-paciente. ${ }^{1-4}$ A palavra empatia deriva do grego empátheia e, segundo dicionário da língua portuguesa, corresponde a experiência pela qual uma pessoa se identifica com outra, tendendo a compreender o que ela pensa e a sentir o que ela sente ainda que, nenhuma das duas, o expressem de modo explícito ou objetivo. ${ }^{5}$

Do ponto de vista clínico, entretanto, o significado preciso de empatia ainda é motivo de discussão. ${ }^{1}$ Os problemas derivam, em parte, de se tratar de construto complexo o qual, no campo da saúde, ainda continua em evolução.

Para Coulehan et $\mathrm{al}^{2}$ empatia, dentro do cenário de atendimento a saúde, constitui a habilidade de entender a situação, perspectivas e sentimentos do paciente e de comunicar aquele entendimento a ele. A ênfase no componente cognitivo (entender a situação), permite o mergulho intelectual nas perspectivas do paciente, não envolvendo o ônus da percepção do sofrimento alheio a partir de uma vivência emocional completa. Desse modo, empatia diz respeito ao entendimento emocional do doente, mantendo-se distância suficiente para que as habilidades médicas possam ser aplicadas racionalmente na solução do problema clínico.

O uso de empatia associa-se a diversos benefícios aos pacientes, entre eles: melhor comunicação de sintomas e preocupações; ${ }^{6-10}$ aumento da acurácia diagnóstica; ${ }^{8,9,11}$ aumento do fornecimento de informações sobre a doença; ${ }^{12-15}$ aumento da participação nas decisões e educação do paciente sobre a doença: ${ }^{14,15}$ aumento da colaboração e satisfação dos pacientes; ${ }^{18,19,20}$ maior aderência ao tratamento, ${ }^{21-25}$ redução da depressão e aumento da qualidade de vida. ${ }^{1,13}$ Já foi igualmente demonstrado que em pacientes com infecções virais das vias aéreas, o grau de empatia do médico associa-se com a duração e gravidade da doença, assim como com variações nos níveis da interleucina- 8 circulante. ${ }^{26} \mathrm{Em}$ pacientes com diabetes mellitus o grau de empatia do médico associa-se com melhor controle da doença e melhora do perfil lipídico. ${ }^{27}$

Em função do exposto, fica claro que o estudo e ensino da empatia para profissionais da saúde se reveste de substancial importância. Para que esses objetivos sejam alcançados, contudo, é fundamental dispormos de instrumentos capazes de avaliar o grau de empatia exibida por esses mesmos profissionais, especialmente estudantes de medicina e médicos.

Diversas escalas foram desenvolvidas visando a avaliação do grau de empatia tais como o Interpersonal Reactivity Index e o Inventário de Empatia, Escala de Tendência Empática. ${ }^{28,29}$ Além disso, existem instrumentos desenvolvidos especificamente para uso no âmbito da saúde, tais como a Barrett-Lennard Empathy Scale, Escala de Empatia de Reynolds e Jefferson Scale of Physician Empathy. ${ }^{30,31,32}$

Uma medida de empatia simples, desenvolvida para emprego na prática médica e que vem sendo utilizada no exterior é denominada CARE (Consultation and Relational Empathy). ${ }^{33,34,35}$ Tal escala foi concebida na Escócia e adequadamente validada para uso em consultórios em nível de atendimento primário. Ela parte do princípio de que o grau de percepção da empatia por parte do paciente influencia a efetividade da intervenção médica. Ou seja, os sentimentos e crenças conscientes do médico não trarão benefícios aos pacientes, a menos que sejam demonstrados de maneira inequívoca. Além disso, se os pacientes são capazes de perceber a quantidade de empatia existente na relação médico-paciente, eles estarão em posição de aconselhar os profissionais sobre como melhorar seu comportamento. Além de partir dessa ótica nova, vantagens adicionais da escala são o número pequeno de itens avaliados e o fato de ser de domínio público.

O presente estudo teve como objetivo traduzir e adaptar a escala CARE do inglês para o português falado no Brasil. Além disso, são fornecidos resultados preliminares relativos à validação do emprego da referida versão em pacientes brasileiros.

\section{Casuística e Métodos}

A versão em inglês da escala CARE foi obtida do artigo original relacionado ao seu desenvolvimento. ${ }^{33}$ Trata-se de instrumento de domínio público, composto por 10 itens, com explicações adicionais de linguagem coloquial visando facilitar a compreensão das perguntas. A graduação é feita com pontuação que varia entre 1 e 5 . Todos os valores dos itens são então somados, fornecendo um escore final entre 10 e 50.

Em um primeiro momento três médicos brasileiros, com profundo conhecimento do inglês e que já haviam vivido pelo menos dois anos em países dessa língua, forneceram as suas traduções da escala. Em 
seguida, um grupo de consenso composto por dois médicos brasileiros, com as mesmas características acima, e dois acadêmicos de curso de medicina, propôs uma versão traduzida inicial. Essa versão sofreu processo de tradução reversa por parte de um professor de inglês, cidadão norte-americano morador no Brasil há décadas. A tradução reversa foi analisada pelo grupo de consenso e, uma vez constatada identidade com a versão original, o processo progrediu. A versão inicial da escala traduzida foi apresentada, de forma preliminar, a alguns pacientes ambulatoriais que relataram dificuldades para compreensão das questões 9 e 10 . Desse modo, o grupo de consenso introduziu alterações nessas questões, visando a adaptação cultural do teste para nossa realidade. Tais adaptações contaram com a concordância do professor de inglês.

Na primeira fase da validação da versão final em português da escala foram incluídos 20 pacientes atendidos no Ambulatório de Pneumologia Geral do Hospital das Clínicas de Ribeirão Preto. Foram selecionados indivíduos adultos, de ambos os sexos, alfabetizados, que não exibiam dificuldades para entendimento ou de comunicação, independente do motivo clínico da consulta. Esses pacientes foram abordados por um de dois pesquisadores (GS, GC), no período de pósconsulta junto ao serviço de enfermagem, e convidados a colaborar com o estudo. Aqueles que aceitavam participar respondiam a versão brasileira da escala CARE, seguida de um conjunto padronizado de perguntas visando avaliação do grau de compreensão da escala. Exemplos de tais questões são: Você entendeu todas as perguntas da escala? Anote abaixo o número das perguntas que você não entendeu.

A segunda etapa do estudo envolveu a aplicação da versão final da escala CARE a 12 pacientes atendidos no mesmo ambulatório, selecionados a partir dos mesmos critérios de inclusão. Os voluntários foram igualmente abordados no momento da pós-consulta e preenchiam um formulário contendo a escala CARE, assim como outras 10 questões componentes de uma Escala de Percepção de Empatia Pelo Paciente (EPEP). Como a aplicação da última escala tinha como finalidade dar substratos de validação concorrente para a escala CARE traduzida, foi necessário selecionarmos um instrumento já disponível em português e que tivesse mostrado propriedades satisfatórias de medição em estudos anteriores. Portanto, neste estudo foi empregada uma versão preliminar da Escala de Jefferson de Percepção de Empatia Pelo Paciente, que já havia sido previamente utilizada, com bons resultados, em pesquisa nacional no campo da obstetrícia. ${ }^{36,37}$ A EPEP é composta por 10 itens, graduados por meio de uma escala Likert entre 1 e 7 , com escores finais variando entre 10 e 70 .

Além disso, nessa segunda fase, os médicos responsáveis pelo atendimento dos referidos 12 pacientes também foram abordados após a consulta, e convidados a responder formulário distinto contendo dois instrumentos genéricos de avaliação de empatia: o Interpersonal Reactivity Index (IRI) e o Inventário de Empatia (IE).

O IRI é um instrumento de avaliação de empatia amplamente utilizado em todo o mundo, tendo já passado por processo de tradução e adaptação transcultural para uso no Brasil. ${ }^{28,38}$ A versão brasileira é composta por 26 questões que levam a formação de 4 subescalas: fantasia, tomada de perspectiva, consideração empática e angústia pessoal. É igualmente possível a obtenção de um escore total de empatia pela soma de todas as subescalas. ${ }^{28}$

O IE é um instrumento de avaliação de empatia desenvolvido no Brasil. ${ }^{29}$ Ele é composto por 40 questões, as quais permitem o estabelecimento de 4 subescalas: tomada de perspectiva, flexibilidade interpessoal, altruísmo e sensibilidade afetiva. É possível ainda a obtenção de um escore total de empatia pela soma das subescalas.

Os resultados do estudo são mostrados na forma de frequências, medianas e intervalos de variação. O grau de consistência interna da escala traduzida foi avaliado pelo cálculo do coeficiente alfa de Cronbach. ${ }^{39}$ Correlações entre os escores da escala CARE e as demais escalas de empatia foram obtidas empregando-se o teste de Spearman. Um valor de p d" 0,05 foi escolhido como limite de significância estatística.

O presente projeto foi aprovado pelo Comitê de Ética em Pesquisas do Hospital das Clínicas de Ribeirão Preto e todos os participantes assinaram termo de consentimento livre e esclarecido.

\section{Resultados}

A versão final em português da escala CARE é mostrada na Tabela 1. Essa escala foi inicialmente aplicada a 20 pacientes (12 mulheres) com mediana de idade de 58 (20-81) anos. Quatorze voluntários informaram 4 anos de escolaridade, dois 8 anos, três ensino médio completo e um ensino superior. Dezenove pacientes informaram que todas as questões eram fáceis de responder. Apenas um referiu dificuldade para entender a questão 10 . Os 20 pacientes referiram que todas as respostas eram fáceis de entender. 


\section{Tabela 1}

Versão brasileira da escala CARE

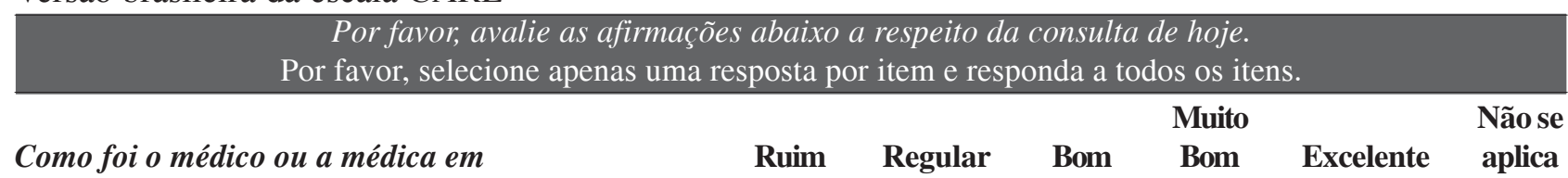

\section{Deixar você à vontade}

(sendo gentil e amigável, tratando você com respeito; sem ser frio(a) ou ríspido(a))

\section{Deixar você contar sua "história"}

(dando tempo para você descrever sua doença completamente, com suas próprias palavras; sem lhe interromper ou distrair)

\section{Realmente ouvir}

(prestando atenção no que você dizia; sem ficar olhando nas anotações ou no computador enquanto você falava)

\section{Estar interessado em sua pessoa como um todo} (perguntando ou sabendo detalhes importantes da sua vida, sua situação; sem lhe tratar "apenas como um número")

\section{Entender plenamente suas preocupações}

(demonstrando que ele ou ela tinha entendido corretamente suas preocupações; não esquecendo ou desconsiderando nada)

\section{Mostrar cuidado e compaixão}

(demonstrando estar genuinamente preocupado, relacionando-se com você em um nível humano; não sendo indiferente ou insensível)

\section{Ser positivo}

(tendo uma abordagem e uma atitude positivas; sendo honesto(a), sem ser negativo(a) sobre os seus problemas)

\section{Explicar as coisas claramente}

(respondendo completamente suas questões, explicando claramente, dando informações adequadas para você; não sendo vago)

\section{Ajudar você a manter o controle}

(conversando sobre o que você pode fazer para melhorar sua saúde; encorajando ao invés de ficar "dando sermão")

\section{Planejar junto com você o que será feito}

(conversando sobre as possibilidades, envolvendo você nas decisões na medida em que você quer estar envolvido(a); não ignorando os seus pontos de vista)

Os valores dos itens individuais são somados fornecendo escore final entre 10 e 50 . Quando houver a opção por "Não se aplica" ou faltar dados, eles podem ser substituídos pela média dos demais valores obtidos. Questionários faltando mais de dois itens ou com mais do que duas respostas "Não se aplica" devem ser desconsiderados. 
Na segunda etapa da investigação, outros 12 pacientes responderam tanto a escala CARE como também a EPEP. O grupo era composto por 7 mulheres e 5 homens com mediana de idade de 44,5 (20-63) anos. O escore mediano da escala CARE para o grupo foi de 41,5 (33-50). O coeficiente alfa de Cronbach calculado a partir das respostas individuais dos 12 pacientes foi 0,867 . Foi encontrada ainda uma correlação estatisticamente significante entre os escores da escala CARE e a escala EPEP (Tabela 2).

Medidas de empatia dos médicos residentes responsáveis pelas consultas foram obtidas para onze indivíduos. O grupo foi composto por 5 homens e 6 mulheres, com mediana de idade de 27 (25-32) anos. Não foram encontradas correlações significantes entre os escores da escala CARE dos pacientes e as pontuações totais da escala IRI ou IE informadas pelos seus respectivos médicos. A única medida de empatia dos médicos que mostrou correlação significante com os escores da escala CARE foi a subescala altruísmo do IE.

\section{Discussão}

O presente artigo relata resultados preliminares de validação de uma versão em português falado no Brasil da escala de empatia CARE. Diante do reconhecimento crescente do valor do construto empatia na relação médico-paciente, e da raridade de estudos semelhantes no campo da medicina brasileira, esta comunicação adquire relevância. Vale salientar ainda que a referida escala é específica para avaliação do grau de empatia por parte de médicos ou estudantes de medicina.

O processo de tradução da escala CARE para o português utilizou metodologia apropriada e os resultados obtidos indicam a validade da versão proposta da escala. ${ }^{40,41}$ Inicialmente, a quase totalidade dos
20 pacientes consultados referiu entendimento adequado sobre as questões e a metodologia envolvida com as respostas. Vale ressaltar que o grupo de entrevistados era composto, na sua maioria, por pessoas de baixa escolaridade, com no máximo 4 anos de estudo, usuários de um hospital público do interior paulista. Portanto, é muito provável que o grau de compreensão da escala deva ser ainda maior em grupos mais intelectualizados.

O coeficiente alfa de Cronbach calculado para as respostas dos 12 voluntários incluídos na segunda etapa da análise foi elevado, indicando que a versão brasileira da escala CARE exibe consistência interna e confiabilidade aceitáveis.

O valor mediano da escala CARE aplicada a 12 pacientes brasileiros foi 41,5 , muito próximo do escore 42 (12-50) observado em 23 pacientes atendidos por médicos do sistema público de saúde escocês incluídos no estudo original de desenvolvimento da escala. ${ }^{33}$ Ainda que não se possa tirar uma conclusão definitiva sobre essa questão, o grau de empatia demonstrado pelos médicos de ambos os serviços parece situar-se em uma faixa satisfatória.

A correlação estatisticamente significante entre a versão brasileira da escala CARE e uma versão preliminar da escala de empatia de Jefferson para pacientes, previamente aplicada em nosso meio a 101 puérperas, é indicador de validade concorrente da nova escala em estudo. Á semelhança da escala CARE o último instrumento reflete as impressões subjetivas do paciente frente ao cuidado recebido pelo profissional da saúde especificamente num contexto assistencial.

A ausência de correlações significativas entre a escala CARE e as medidas de empatia global IRI e IE dos médicos assistentes é constatação importante. Esse achado não invalida a utilidade da escala CARE, uma vez que ela se baseia exclusivamente no ponto

\section{Tabela 2}

Medidas de correlação entre a escala CARE e outros índices de empatia

\begin{tabular}{cc|ccccc|ccccc}
\hline & & \multicolumn{4}{|c||}{ Interpersonal Reactivity Index } & \multicolumn{4}{c}{ Inventário de Empatia } \\
\cline { 3 - 12 } & \multirow{2}{*}{ EPEP $^{+, \#}$} & Fantasia & $\begin{array}{c}\text { Tomada de } \\
\text { Perspectiva }\end{array}$ & $\begin{array}{c}\text { Consideração } \\
\text { Empática }\end{array}$ & $\begin{array}{c}\text { Angústia } \\
\text { Pessoal }\end{array}$ & $\begin{array}{c}\text { Escore } \\
\text { Total }\end{array}$ & $\begin{array}{c}\text { Tomada de } \\
\text { Perspectiva }\end{array}$ & $\begin{array}{c}\text { Flexibilidade } \\
\text { interpessoal }\end{array}$ & $\begin{array}{c}\text { Altruísmo } \\
\text { Sensibilidade } \\
\text { Afetiva }\end{array}$ & $\begin{array}{c}\text { Escore } \\
\text { Total }\end{array}$ \\
\hline $\mathrm{r}^{*}$ & 0,70 & $-0,42$ & $-0,24$ & 0,11 & 0,53 & $-0,06$ & $-0,01$ & 0,19 & 0,74 & 0,15 & 0,23 \\
$\mathrm{p}$ & 0,01 & 0,19 & 0,47 & 0,75 & 0,09 & 0,86 & 0,99 & 0,58 & 0,01 & 0,67 & 0,49 \\
\hline
\end{tabular}

* Coeficiente de correlação de Spearman.

+ Escala de percepção de empatia pelo paciente.

\# Correlação envolvendo 12 pares de medidas; demais correlações com $n=11$ 
de vista dos pacientes. Entretanto, é indicação que mesmo profissionais com graus elevados de empatia exibem dificuldades para transmitir satisfatoriamente tal experiência aos pacientes. Portanto, muitos médicos que hoje acreditam estar desempenhando papel satisfatório junto aos seus clientes podem, na realidade, estar falhando substancialmente nesse aspecto da comunicação com os pacientes. Além disso, não podemos excluir a possibilidade que fatores externos à relação médico-paciente possam ter influenciado tais resultados. Assim, por exemplo, um número excessivo de pacientes a serem atendidos, bem como dificuldades de acesso a resultados de exames subsidiários, pode ter dificultado a exteriorização de aspectos humanísticos do profissional. Ou seja, é possível que condições de trabalho adversas dificultem a expressão de empatia por parte dos médicos.

A única correlação entre uma medida de empatia dos médicos, com os escores CARE dos pacientes, que mostrou significância estatística, envolveu a subescala altruísmo do IE. Essa subescala reflete um componente afetivo da empatia, relacionado ao grau de consideração exibido pelas necessidades de outros seres humanos, e a tendência a agir em conformidade com tais necessidades. ${ }^{42}$ Devido a sua natureza afetiva, é de se supor que o altruísmo seja em parte dependente de características psicológicas e emocionais inatas dos indivíduos. ${ }^{43,44}$ Apesar disso, dados recentes indicam que os atributos emocionais de estudantes de medicina podem ser aprimorados, mesmo após intervenções relativamente curtas. ${ }^{45}$ Desse modo, os resultados aqui obtidos sugerem que o ensino e treinamento de altruísmo a estudantes de medicina possam ser investigados como formas de aprimoramento da empatia dos futuros médicos.
O presente estudo exibe uma série de limitações, a maior crítica sendo o número pequeno de pacientes e médicos incluídos na investigação. Contudo, vale salientar que mesmo o trabalho original, relacionado ao desenvolvimento da escala CARE, envolveu um número pequeno de pacientes na etapa de validação da versão final do instrumento, 13 e 10 voluntários nos estudos piloto 2 e 3 respectivamente. Uma grande dificuldade para o desenvolvimento deste trabalho foi obter a colaboração dos médicos responsáveis pelas consultas dos pacientes. Seja por sobrecarga de trabalho, seja por resistência à participação em uma investigação ligada a aspectos emocionais da comunicação médico-paciente, boa parte dos profissionais demorou para entregar suas fichas preenchidas e um deles nunca o fez.

\section{Conclusão}

Em conclusão, podemos afirmar que a versão em português falado no Brasil da escala CARE é de fácil compreensão e uso por usuários do sistema público de saúde. Alem disso, exibe evidências de validação concorrente aceitáveis. Trata-se, portanto, de instrumento adequado para o desenvolvimento de novos estudos relacionados com o tema da empatia no contexto médico em nosso meio. $\mathrm{O}$ fato das medidas de empatia referidas pelos médicos tenderem a não se correlacionar com as percepções dos pacientes merece estudos adicionais.

\section{Agradecimentos}

Os autores agradecem a Rodrigo Tocantins Calado e Dennis Farquharson pela colaboração com este trabalho.

\footnotetext{
ABSTRACT

Objectives: To translate and to adapt the scale Consultation and Relational Empathy (CARE) for using in Brazil. To obtain preliminary results about concurrent validation of this scale.

Methods: The scale was translated and adapted from English by appropriated recommended methodology. Twenty patients from the pulmonology clinic of a public hospital, associated with the Brazilian public health system, were interviewed about the degree of understanding of the scale final version. The second step of the study enrolled twelve patients from the same clinic who answered the Brazilian version of CARE and the Perception Scale of Empathy By Patients (EPEP), shortly after the end of a routine consultation The physicians responsible for the care of these patients were also approached and asked to answer a questionnaire containing the Brazilian version of the Interpersonal Reactivity Index (IRI) and the Inventory of Empathy (IE).

Results: Nineteen of 20 patients reported a high degree of understanding about the items of the Brazilian version of CARE. The median CARE score for other 12 volunteers was 44.5 (20-63). The Cronbach's alpha coefficient for the answers of these patients was 0.867 . CARE scores significantly correlated with
} 
EPEP scores $(r=0.699, p=0.01)$ and with subsection altruism of the IE $(r=0.744, p=0.01)$. CARE scale showed no significant correlations with global scores of IE and IRI reported by physicians.

Conclusion: The Brazilian version of CARE is easy to understand and displays acceptable evidences of concurrent validity and internal consistency. Measures of empathy reported by doctors tend to not correlate with perceptions of patients.

Keywords: Empathy. Psychology, Medical. Relative Value Scales. Translating.

\section{Referênclas}

1. Mercer SW, Reynolds WJ. Empathy and quality of care. Br J Gen Pract. 2002; 52: S9-S12.

2. Coulehan JL, Platt FW, Egener B, Frankel R, Lin CT, Lown B et al. "Let me see if I have this right...": Words that help build empathy. Ann Intern Med. 2001; 135: 221-7.

3. Neumann M, Bensing J, Mercer S, Ernstmann N, Ommen O, Pfaff $\mathrm{H}$. Analyzing the "nature" and "specific effectiveness" of clinician empathy: A theoretical overview and contribution towards a theory-based research agenda. Patient Educ Couns. 2009; 74: 339-46.

4. Neumann M, Scheffer C, Tauschel D, Lutz G, Wirtz M, Edelhäuser F. Physician empathy: definition, outcome-relevance and its measurement in patients care and medical education. GMS Z Med Ausbild. 2012; 29(1): Doc11.

5. Dicionário Aulete online. http://aulete.uol.com.br/ site.php?mdl=aulete_digital. Acessado em 26/04/2012.

6. Squier RW. A model of empathic understanding and adherence to treatment regimens in practitioner-patient-relationships. Soc Sci Med. 1990;30: 325-9.

7. Stepien KA, Baernstein A. Educating for empathy. J Gen Intern Med. 2006; 21: 524-30.

8. Maguire $P$, Faulkner $A$, Booth $K$, et al. Helping cancer patients disclose their concerns. Eur J Cancer Care. 1996; 32A: 7881.

9. Beckman HB, Frankel RM. Training practitioners to communicate effectively in cancer care: it is the relationship that counts. Patient Educ Couns. 2003; 50: 85-9.

10. Eumann M, Wirtz M, Bollschweiler E, Warm M, Wolf J, Pfaff H. Psychometrische Evaluation der deutschen Version des Messinstruments "Consultation and Relational Empathy" (CARE) am Beispiel von Krebspatienten. Psychother Psychosom Med Psychol. 2008; 58: 5-15.

11. Larson EB, Yao X. Clinical empathy as emotional labor in the patient-physician relationship. JAMA. 2005; 293: 1100-6.

12. Irving P, Dickson D. Empathy: towards a conceptual framework for health professionals. Int J Health Care Qual Assur Inc Leadersh Health Serv. 2004; 17: 212-20.

13. Neumann M, Wirtz M, Bollschweiler E, Mercer SW, Warm M, Wolf $\mathrm{J}$, et al. Determinants and patient-reported long-term outcomes of physician empathy in oncology: A structural equation modeling approach. Patient Educ Couns. 2007; 69: 63-75.

14. Neumann M, Bensing J, Mercer S, Ernstmann N, Ommen O, Pfaff H. Analyzing the "nature" and "specific effectiveness" of clinician empathy: A theoretical overview and contribution towards a theory-based research agenda. Patient Educ Couns. 2009; 74: 339-46.

15. Kim SS, Kaplowitz S, Johnston MV. The effects of physician empathy on patient satisfaction and compliance. Eval Health Prof. 2004; 27: 237-51.
16. Price S, Mercer SW, MacPherson H. Practitioner empathy, patient enablement and health outcomes: A prospective study of acupuncture patients. Patient Educ Couns. 2006; 63: 23945.

17. Roter DL, Stewart M, Putnam SM, Lipkin M Jr, Stiles W, Inui TS. Communication patterns of primary care physicians. JAMA. 1997; 277: 350-6.

18. Nightingale SD, Yarnold PR, Greenberg MS. Sympathy, empathy, and physician responses in primary care and surgical settings. J Gen Intern Med. 1991;6: 420-3.

19. Levinson W, Gorawa-Bhat R, Lamb J. A study of patient cues and physician responses in primary care and surgical settings. JAMA. 2000; 284: 1021-7.

20. Howie JGR, Heaney DJ, Maxwell MW, et al. Quality at general practice consultations: cross sectional survey. $\mathrm{Br}$ Med $\mathrm{J}$ 1999;319: 738-43.

21. MacPherson H, Mercer SW, Scullion T, Thomas KJ. Empathy, enablement, and outcome. J Altern Complement Med. 2003;9: 869-76.

22. Bikker AP, Mercer SW, Reilly D. A pilot prospective study on the consultation and relational empathy, patient enablement, and health changes over 12 months in patients going to the Glasgow Homoeopathic Hospital. J Altern Complement Med. 2005;11: 591-600.

23. Mercer SW, Reilly D, Watt GC. The importance of empathy in the enablement of patients attending the Glasgow Homoeopathic Hospital. Br J Gen Pract. 2002; 52: 901-5.

24. Mercer SW, Watt GC, Reilly D. Empathy is important for enablement. BMJ. 2001; 322: 865.

25. Mercer SW, Neumann M, Wirtz M, Fitzpatrick B, Vojt G. General practitioner empathy, patient enablement, and patientreported outcomes in primary care in an area of high socioeconomic deprivation in Scotland: a pilot prospective study using structural equation modeling. Patient Educ Couns. 2008; 73: 240-5.

26. Rakel DP, Hoeft TJ, Barrett BP, Chewning BA, Craig BM, Niu $\mathrm{M}$. Practitioner empathy and the duration of the common cold. Fam Med. 2009; 41: 494-501.

27. Hojat M, Louis DZ, Markham FW, Wender R, Rabinowitz C, Gonnella JS. Physicians' empathy and clinical outcomes for diabetic patients. Acad Med. 2011; 86: 359-64.

28. Davis, Mark H. Measuring individual differences in empathy: Evidence for a multidimensional approach. J Pers Soc Psychol. 1983; 44: 113-26.

29. Falcone E, Ferreira M, Luz R, Fernández C, Faria C, D'Augustin $\mathrm{J}$, et al. Inventário de Empatia (IE): desenvolvimento e validação de uma medida brasileira. Aval Psicol. 2008; 7: 321-34.

30. Layton JM, Wykle MH. A validity study of four empathy instruments. Res Nurs Health. 1990;13: 319-25.

31. Reynolds B. The influence of clients' perceptions of the helping relationship in the development of an empathy scale. $J$ Psychiatr Ment Health Nurs. 1994; 1: 23-30. 
32. Hojat M, Mangione S, Nasca TJ, Cohen MJ, Gonnella JS, Erdmann JB, et al. The Jefferson scale of empathy: development and preliminary psychometric data. Educ Psychol Measur. 2001; 61:349-65.

33. Mercer SW, Maxwell M, Heaney D, Watt GC. The consultation and relational empathy (CARE) measure: development and preliminary validation and reliability of an empathy-based consultation process measure. Fam Pract. 2004; 21: 699705.

34. Mercer SW, McConnachie A, Maxwell M, Heaney D, Watt GC. Relevance and practical use of the Consultation and Relational Empathy (CARE) Measure in general practice. Fam Pract. 2005; 22: 328-34.

35. Wirtz M, Boecker M, Forkmann T, Neumann M. Evaluation of the "Consultation and Relational Empathy" (CARE) measure by means of Rasch-analysis at the example of cancer patients. Patient Educ Couns. 2011; 82: 298-306.

36. Lima SP. O cuidado humanístico como foco institucional: um estudo sobre empatia dos profissionais de saúde na área obstétrica. Dissertação de mestrado, 2004, Universidade Federal do Rio Grande do Norte.

37. Kane GC, Gotto JL, Mangione S, West S, Hojat M. Jefferson Scale of Patient's Perceptions of Physician Empathy: preliminary psychometric data. Croat Med J. 2007; 48: 81-6.
38. Sampaio LR, Guimarães PRB, Camino CPS, Formiga NS, Menezes IG. Estudos sobre a dimensionalidade da empatia: tradução e adaptação do Interpessoal Reactivity Index (IRI). Psico. 2011; 42: 67-76.

39. Schmitt N. Uses and abuses of coefficient alpha. Psychol Assess. 1996; 8:350-3.

40. Hambleton RK. Translating achievement tests for use in crossnational studies. Eur J Psychol Assess. 1993; 9: 57-68.

41. Geisinger KF. Cross-cultural normative assessment: translation and psychological and adaptation issues influencing the normative interpretation of assessment instruments. Psychol Assess. 1994; 6: 304-12.

42. Batson CD. Self-other merging and the empathy-altruism hypothesis: Reply to Neuberg et al. (1997). J Pers Soc Psychol. 1997; 73: 517-22.

43. Rachlin H, Locey M. A behavioral analysis of altruism. Behav Process. 2011; 87: 25-33.

44. Aknin LB, Hamlin JK, Dunn EW. Giving leads to happiness in young children. PLos ONE . 2012;7: e39211.

45. Abe K, Evans P, Austin EJ, Suzuki Y, Fujisaki K, Niwa M, et al. Expressing one's feelings and listening to others increases emotional intelligence: a pilot study of Asian medical students. BMC Med Educ. 2013;13: 82. 